Article

\title{
Room Classification Based on EMC Conditions in Nuclear Power Plants
}

\author{
Hrvoje Grganić ${ }^{1, *}$, Davor Grgić ${ }^{2}$ and Siniša Šadek ${ }^{2}$ (D) \\ 1 Krško Nuclear Power Plant, Vrbina 12, Krško 8270, Slovenia \\ 2 Faculty of Electrical Engineering and Computing, University of Zagreb, Unska 3, Zagreb 10000, Croatia; \\ davor.grgic@fer.hr (D.G.); sinisa.sadek@fer.hr (S.Š.) \\ * Correspondence: hrvoje.grganic@nek.si; Tel.: +385-99-708-4794
}

Received: 3 December 2019; Accepted: 9 January 2020; Published: 11 January 2020

\begin{abstract}
Electromagnetic compatibility (EMC) in nuclear power plants today mostly relies on the qualification tests of the new equipment and adhering to some good installation practices. Diversity of the electromagnetic environment and different susceptibility of the plant equipment calls for a systematic classification of the EMC zones in a nuclear power plant. The paper proposes a methodology that uses a combination of the qualification tests, in situ and bench immunity tests, site survey measurements, operational experience, and numerical calculations to divide a nuclear power plant into a reasonable number of EMC zones. This would primarily help to have a better overview of the current EMC level in the plant and to unify emission and susceptibility requirements for the new equipment. In this paper, special attention is given to the preparation and performance of the in situ tests, which present the most challenging step of the methodology. In addition, the paper proposes some of the possible applications of the numerical calculations and addresses their challenges and limitations. The novel classification methodology, inspired by the equipment qualification program, is illustrated with examples from Krško Nuclear Power Plant, which recently performed a comprehensive EMC assessment.
\end{abstract}

Keywords: electromagnetic compatibility; electromagnetic immunity; electromagnetic fields; in situ tests; nuclear power plants

\section{Introduction}

The electromagnetic compatibility (EMC) problem has been increasingly recognized in the nuclear industry in the past decades as many of the power plants have performed digital upgrades, thereby replacing the original analogue instrumentation and control (I\&C) equipment with the more susceptible hybrid and digital I\&C equipment [1]. Nuclear power plants (NPPs) are in many ways specific from the electromagnetic compatibility point of view. In addition to the above-mentioned ongoing process of digital upgrades, they are characterized by a variety of I\&C equipment that are important for safety, which is susceptible to electromagnetic (EM) fields in different ways. NPPs also usually have a highly diverse electromagnetic environment (EME).

There are numerous sources of EM noise in a typical power plant such as fault current clearance by switchgear, circuit breaker, or fuse operation and the operation of motors, generators, transformers, inverters, rectifiers, switch-mode power supply, relays, solenoids, and other emitters.

On the other hand, in a typical NPP, there are cabinets that process vital plant signals, different pressure, temperature, flow, and other instrumentation as well as other sensitive electronics operating with extremely low signals such as radiation monitors.

For these reasons, it is not easy to obtain an overall view of the plant's EMC, which inevitably leads to problems with the definitions of the EMC requirements for the new equipment and the exclusion 
zones, implementation of the wireless technologies, troubleshooting of the EMC-related problems, and others. The IEC standard 61000-2-5 [2] presents one of the very few attempts for the classification of EM environments, with the intention to identify a limited set of parameters and associated values that may be chosen when identifying performance requirements.

EMC regulatory requirements for I\&C equipment were either not developed or not in effect until the last few years. Therefore, there is a considerable number of equipment in NPPs that have not been qualified for EMC. The current EMC regulatory requirements only address new and modified equipment, and do not call for the testing of existing equipment. There is a gap that has to be overcome in order to understand the actual level of EMC in the plant.

There is no clear, detailed, and unambiguous guidance on how to perform any of these tests on the already installed equipment. Some nuclear power plants have undergone similar tests, although these were less extensive compared to the Krško NPP [3-5]. It takes a lot of engineering judgement to optimize tests for a specific plant. Some of the most important questions addressed in this paper are: (1) the selection of the plant areas for site survey measurements and susceptible equipment to be tested for immunity; (2) selection of the representative electromagnetic disturbances that shall be simulated during those tests; and (3) practical performance (i.e., coordination of immunity tests with operation of other plant systems and minimization of the undesired interference). Site survey guidance can be partially found in several papers [6], which are mostly outdated, as well as IEEE Std. 473-1985, which has been withdrawn.

The paper proposes a methodology that uses a combination of the qualification tests, in situ and bench immunity tests, site survey measurements, operational experience, and numerical calculations to divide a nuclear power plant into a reasonable number of EMC zones.

The idea for the methodology presented in this paper was derived from similar zone classifications used in an environmental qualification (EQ) plant program. EQ originated as a consequence of the Three Mile Island accident. The purpose of the development of EQ zone maps and conditions is to demonstrate the capability of nuclear safety-related (NSR) equipment to perform its safety function in aged conditions and under extreme conditions after a design basis event [7]. Equipment qualification is also one of the steps of the nuclear licensing process. The principal environmental conditions typically addressed for both normal and accident conditions are: temperature, pressure, relative humidity, chemical spray, submergence, radiation, and vibration and seismic motion.

Environmental zones are defined based on the EQ parameter values during normal, but also abnormal, accident, and post-accident conditions (i.e., it includes harsh accident environment following loss-of-coolant accident (LOCA), high energy line break (HELB), and others). The normal condition can also be determined by in situ measurements. Each EQ zone has a unique identification number, similar values of the relevant EQ parameters, and well-defined boundaries. The zones may consist of an entire building, but typically consist of one or more building compartments. The EQ zone is a way of specifying global environmental conditions for all equipment located inside an EQ zone.

EQ zone maps are prepared by marking the boundaries of such areas on the plant layout drawings. There is no set methodology for preparing EQ zones and EQ maps, as this process is plant specific and should involve engineering judgement in assigning environmental conditions to the plant's physical layout.

Unlike the environmental qualification zones, for which there is a strong correlation between those conditions and the common-cause failures, the outcome of electromagnetic interference (EMI) is less predictable and also less likely to affect multiple equipment at once [8]. The parameters characterizing the electromagnetic environment are present in normal operation and are not necessarily associated with any design basis event. Taking that into account, we are not expecting that EMC room classification will end in a simple harsh/mild form like the case in EQ classification. It is more likely to obtain several categories describing the room from a point of view of susceptible equipment presence and/or appearance of the excessive EM parameters. 


\section{Classification Methodology}

The combination of the operating records, in situ and bench immunity tests, site survey measurements, and numerical calculations can provide a reasonable amount of data to perform a systematic NPP room classification based on EMC conditions. Each of these inputs contributes in a specific way, as discussed below.

Historical data can help identify some of the key plant problems, which can later be confirmed through immunity tests, which could provide more detailed information about the susceptibility problems. Operating records can also help to define the exact scope of the immunity tests.

Site survey measurements can help to compare EM field levels in different areas of the plant, and can identify those with increased emissions. It is not only possible to recognize that some rooms have higher emissions, but also to precisely characterize the noise.

Site survey results are partially confirmed by and extended with numerical calculations, as it is not possible to perform measurements in a large number of plant locations. Numerical calculations of the cabinet shielding also provide useful information regarding the field attenuation, providing inputs for the design of the exclusion zones (i.e., definition of the exclusion distance from the susceptible equipment). They can also give information at which distance from the emitter the field values drop down to the acceptable levels, and predict improvements based on the design alterations.

The zone classification methodology flowchart is shown in Figure 1. The methodology starts with the two processes that can be performed simultaneously.

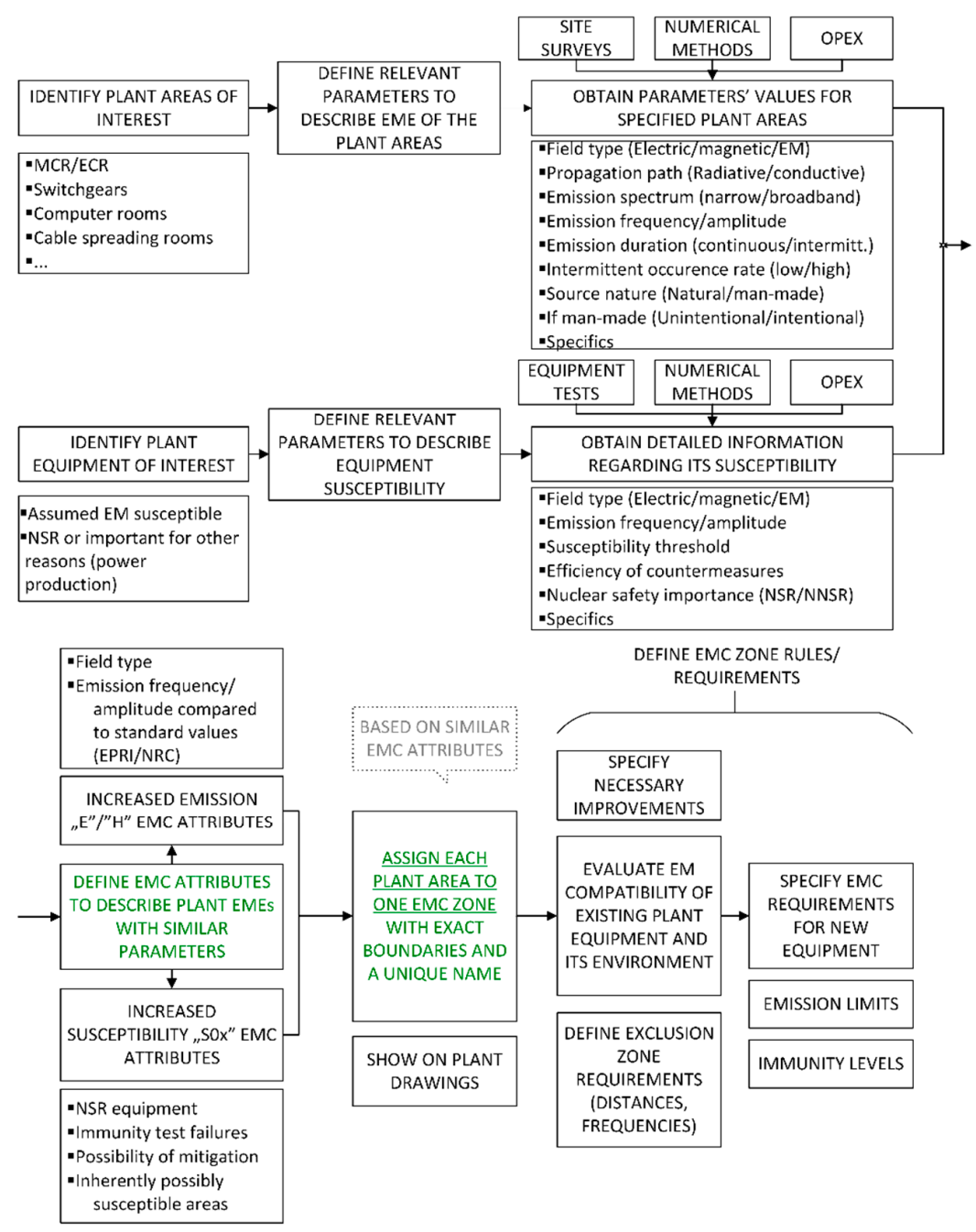

Figure 1. EMC zone classification methodology flowchart. 
The first process is related to the characterization of the plant areas from the EMC point of view. In order to perform that task, first, it is necessary to identify plant areas of the interest (i.e., all areas where either potential emitters are located, susceptible equipment is located, or which serve as the interconnection of the different equipment). This step will slightly reduce the scope of the observed areas and help focus on the important ones. Afterward, we proceed by defining relevant parameters to describe the EME of the plant areas, which should:

a. describe plant areas with a sufficient amount of information regarding its EME;

b. be (easily) obtainable, either from the site surveys, numerical calculations or operating experience;

c. be measurable and unambiguous; and

d. be relevant for the assessment of the susceptibility of the equipment located in the observed areas.

Such a set of parameters can include the dominant field type (electric, magnetic), propagation path (radiated, conducted), emission spectrum (narrowband or broadband), emission frequencies and amplitudes, emission duration (transient or continuous), and nature (natural, man-made: unintentional or intentional). The parameters are obtained through a combination of previously discussed techniques.

The second part of the process is related to the characterization of the susceptible equipment. It is done in a similar manner as for the plant areas and it starts with the identification of the potentially susceptible equipment. The next steps are also similar. It is necessary to define the relevant parameters that can describe the susceptibility (immunity) of the I\&C equipment and obtain the values of those parameters. The choice of parameters is similar to that discussed for EME characterization.

From here on, it is necessary to analyze the outcomes of both processes and merge the results. Instead of trying to establish strict quantitative criteria for the EMC zones, we prefer to define them more descriptively. In order to be consistent with the first part of the methodology, two EMC attribute types are proposed: "S" zone attributes that will define the susceptibility level of the equipment, and " $\mathrm{E} / \mathrm{H}$ " zone attributes that will define the properties of the EME, because each plant area can be characterized at the same time with both susceptibility and emission attributes. It is critical to both recognize that the excessive emissions could affect potentially susceptible equipment as well as the opposite case, where we have already identified that there is susceptible equipment, which means that the emissions should be strictly controlled. EMC attributes are an intermediate step of this methodology, which helps to group the EMEs with similar characteristics into EMC zones. The used attributes are described below, with the definition flowchart used to assign them given in Figure 2.

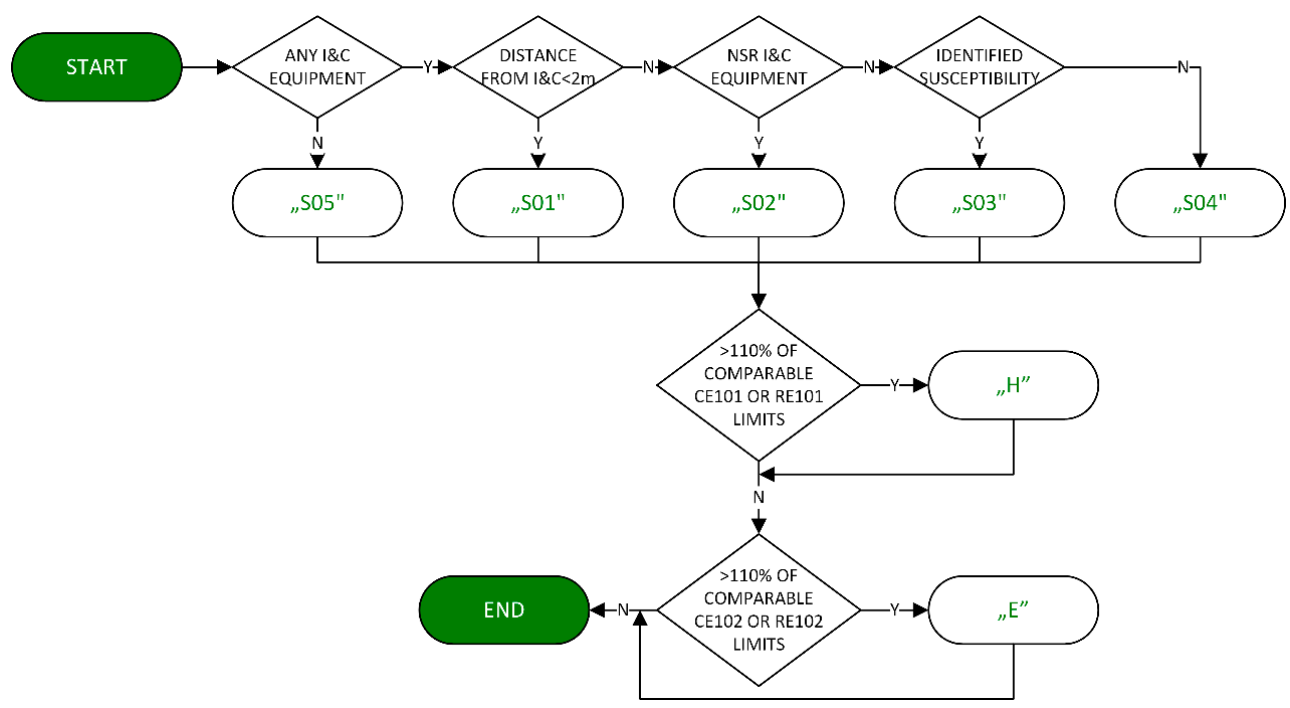

Figure 2. Flowchart assigning the susceptibility and emission attributes. 
Finally, the locations with similar EMC attributes are called an EMC zone. The EMC zone has well-defined boundaries shown on the plant layout drawings and a unique identification number, similar to that undertaken for the previously mentioned EQ methodology.

\section{EMC Attributes}

Immunity tests and numerical calculations have proven that the use of communication devices is not allowed under any conditions for most of the operating cabinets with the doors open. Taking that into account, all susceptibility attributes are assigned to the configuration where the cabinet doors are closed.

\section{Susceptibility attribute 1 (S01)}

Special attention must be paid to the area near the sensitive plant equipment. The S01 zone attribute primarily encompasses a distance up to two meters from all nuclear safety-related plant cabinets and I\&C instrumentation. It is set for all emitters, regardless of the emission frequency, since it is not related to an identified vulnerability, but to the fact that the possibility for interference rapidly increases for smaller distances between the emitter and victim.

The above-mentioned distance of $2 \mathrm{~m}$ was obtained in the following way. Immunity tests were performed using a predefined electric field strength, which was $10 \mathrm{~V} / \mathrm{m}$ or higher for the selected equipment under test (EUT), as defined by the regulatory requirements. If the EUT successfully passed the test, the exclusion zone around it was defined. First, a safety margin of $8 \mathrm{~dB}$ was used to eliminate uncertainties related to the EME and address that it is always possible to expose the equipment to higher field values than it was tested for. The emitter output power might be higher, or the distance to the plant equipment might be smaller. If the test E-field strength is $10 \mathrm{~V} / \mathrm{m}$, the allowed E-field strength is $4 \mathrm{~V} / \mathrm{m}$ in this case.

It is possible to estimate the exclusion zone around the tested equipment using the equation below [9]. The equation relates the maximum allowed strength of the electric field $E(\mathrm{in} \mathrm{V} / \mathrm{m})$, the measured output power of the test system $P_{t}$ (in watts), and the test antenna gain $G_{t}$ (dimensionless), with the minimum exclusion zone distance $D$ (in meters).

$$
D=\frac{\sqrt{30 P_{t} G_{t}}}{E}
$$

For typical values of $P_{t}$ and $G_{t}$ for the plant radios, the calculated exclusion distance was $1.37 \mathrm{~m}$. We performed immunity tests on plant components using appropriate E-field strengths. Then, for different emitters such as plant radios, mobile phones, and Digital Enhanced Cordless Telecommunications (DECT) phones, we calculated the minimum exclusion distances. None of the calculated distances exceeded $2 \mathrm{~m}$. Therefore, this value was taken as the unique exclusion zone for all I\&C equipment.

Although it could be obviously relaxed for some equipment, it is more practical to define a unique exclusion zone for all equipment (which is here conservative compared with the minimum recommendations given by EPRI-1/3 m), instead of defining it for each piece of equipment separately. The proposed distance is acceptable as a compromise between achieving EMC immunity and not limiting plant personnel at the same time. For some cases, it is reduced several times from what was previously defined as a minimum exclusion zone (often, the whole plant rooms or at least significantly larger distances from the susceptible equipment are excluded).

Numerical calculations (field spatial distribution examples) and empirical formulas confirmed that the field attenuation is in accordance with Equation (1). It is known that both the electric field and the magnetic field attenuate rapidly $\left(1 / \mathrm{r}^{3}\right.$ and $1 / \mathrm{r}^{2}$, respectively) in the near-field region, and $1 / \mathrm{r}$ terms begin to dominate in the equation for the field intensity in the far-field region. For example, the far-field region typically begins $10-20 \mathrm{~cm}$ from the plant radios, depending on their operating frequency. Hence, it is critical to prevent the use of emitters in the proximity of the I\&C plant equipment. 
Only a fraction of all of the plant I\&C equipment was tested. Hence, although the tested equipment might be representative, one must be aware of the limitations of such assessment. The exclusion distance could also be larger if some equipment did not pass some of the immunity tests.

In addition to the intentional emitters that are usually controlled administratively, it is necessary to strictly control and minimize the use of the unintentional emitters, which could easily affect the susceptible equipment. This implies the use of higher qualification emission requirements for the newly installed equipment, particularly RS103 and the corresponding IEC EN61000-4-3 [10] tests. However, the first option is always to find a more appropriate installation location for such equipment. Every modification of the existing NSR I\&C cabinets should be evaluated from the EMC point of view. It is recommended that universal immunity improvement measures are applied, which are valid for all installations such as the use of fine meshes to minimize enclosure openings and eliminate non-metallic enclosures.

2. Susceptibility attribute 2 (S02)

The S02 susceptibility zone attribute is assigned to plant areas where the susceptible or potentially susceptible nuclear safety-related I\&C equipment was identified. Identification of these areas is strongly supported by in situ immunity tests.

Unlike the S01 zone attribute, the restrictions for the S02 zone attribute only apply to frequencies below $1 \mathrm{GHz}$, mostly based on the results of the immunity assessment.

Again, no severe emitters should be installed in these areas, and modification should include a comprehensive EMC review. Immunity requirements should be on the same level as for the S01.

The central, and from the EMC point of view, the most important area of the plant, is the main control room (MCR), followed by its counterpart the emergency control room (ECR) as well as the plant computer rooms. There, the most important instrumentation readings and controls are wired. Other specific areas are the cable spreading rooms. These are two separated plant areas, one for control cables, and the other for the power cables, through which most of the plant cables are routed. Although almost no equipment is placed there, cables can easily pick up the EM noise and reradiate it, causing unpredictable interference in other, remote plant areas. While administrative restrictions related to the use of the plant radios and similar devices can affect communication efficiency of the plant personnel in other plant areas, it might be an acceptable and efficient solution for cable spreading rooms.

3. Susceptibility attribute 3 (S03)

Another attribute, designated as $\mathrm{S} 03$, relates to the rooms where the susceptible non-nuclear safety related (NNSR) equipment were identified. The EMC attribute was assigned even if an acceptable solution to mitigate or completely cancel interference by modifying the EUT design or installation was found.

Identification of these areas is also strongly supported by in situ immunity tests.

The first step to improve the equipment immunity in those areas is by applying a simple design and installation enhancements, some of which like shielding effectiveness can be confirmed with numerical calculations. Afterward, the requirements for the new equipment should be defined. It is reasonable to use stricter emission requirements when qualifying this equipment. These increased requirements should be limited to the frequencies for which the susceptibility is identified. It is defined as an exclusion zone for the specific frequencies of interest (i.e., for the specific emitters only, usually plant radios or mobile phones).

\section{Susceptibility attribute 4 (S04)}

The most relaxed requirements are defined for plant areas where no immunity problems have been detected and no nuclear safety-related equipment is located. These are designated as S04.

As already said, there are no regulatory requirements for the immunity of the NNSR equipment.

However, in order to minimize the nuisance of the plant personnel caused by false alarms or readings, it is recommended to keep the equipment immunity at least reasonably high compared to 
the rest of the plant. This is the candidate area for the installation of the largest emitters, as long as the emissions are contained by using line filters, shielding, and similar. No exclusion zone rules apply for these plant areas.

For example, a large part of the turbine building could be characterized as such.

5. Susceptibility attribute 5 (S05)

The last zone encompasses plant areas where no I\&C equipment is located, or is very limited and not important for nuclear safety. Such areas mostly contain piping or mechanical components with no instrumentation. These areas could be designated and treated as "RF safe areas", as similarly described in EPRI TR-102323 [9]. No exclusion zone rules nor any restrictions apply for this zone.

A brief summary of the above described susceptibility zone attributes is given in Table 1 .

Table 1. Summary of susceptibility zone attributes S01-S05.

\begin{tabular}{cccccc}
\hline Attribute & $\begin{array}{c}\text { Safety Class } \\
\text { Equipment }\end{array}$ & $\begin{array}{c}\text { Susceptibility } \\
\text { Identified }\end{array}$ & $\begin{array}{c}\text { Exclusion Zone } \\
\text { Requirements }\end{array}$ & $\begin{array}{c}\text { Presence of I\&C } \\
\text { Equipment }\end{array}$ & Boundaries \\
\hline S01 & NSR & Not necessarily & All frequencies & Yes & $\begin{array}{c}\text { Vicinity of } \\
\text { equipment }\end{array}$ \\
\hline S02 & NSR & Yes & $\begin{array}{c}<\mathrm{GHz} \\
\text { Frequencies of } \\
\text { susceptibility }\end{array}$ & Yes & Yes \\
S03 & NNSR & Yes & None & Yes & boundarical \\
S04 & NNSR & No & None & No & \\
\hline
\end{tabular}

6. E-field emission attribute (E)

The site survey identified several plant areas that had higher than average electric field emission levels. The criterion for increased emissions was set to approximately $10 \%$ above the comparable regulatory emissions limits. As the site survey provided field values for only a limited number of discrete locations, it was necessary to extrapolate the results for the rest of the area by approximating it with the field propagation equations and verifying them with the numerical models. The size of the increased emission areas was defined so that the field values on the zone boundary were approximately $20 \%$ below the comparable emissions limits.

Unlike the magnetic field, it was proven and shown in this paper that the E-field emissions interfere with different I\&C equipment. In order to encounter this kind of interference, it is necessary to have an E-field source, which is a high impedance (high voltage, low current) source, and electrically small dipoles that can pick-up E-fields.

It is generally recommended to avoid these locations for the installation of new equipment if possible. If this is not possible, at least it is necessary to qualify the equipment for higher immunity levels (RS103 and corresponding IEC EN61000-4-3 [10] tests). It is also recommended that tests are extended for the new and existing equipment above $1 \mathrm{GHz}$ (up to $10 \mathrm{GHz}$ ), even though this might not be requested by some standards. For this purpose, in situ or bench tests could be performed, or the present E-field emissions could be analyzed, in order to prove that they do not pose a threat.

7. H-field emission attribute $(\mathrm{H})$

There are plant areas where the values of the H-field are significantly increased, similar as described for the E-fields. Very little equipment in the plant is susceptible to magnetic fields, and this type of interference is much more important for some other applications such as the navy (low frequency acoustic systems and extremely low frequency (ELF) to LF communication systems and sensors). In order to encounter this kind of interference, it is necessary to have an H-field source, which is a low impedance (low voltage, high current) source and electrically small loop antennas that can pick-up H-fields. Some examples of susceptible equipment are cathode ray tubes and Hall Effect sensors. The latter are used for proximity sensing, positioning, speed detection, and they have 
an output voltage that is directly proportional to the magnetic field strength through it. Except for the mentioned equipment, it is possible to relax installation restrictions in areas with high magnetic fields, which are quite often in the plant. These are usually in the vicinity of the high current carrying conductors and power electronics. RE101 plant measurements have confirmed that these can be found in the vicinity of uninterruptible power supplies (UPSes) and diesel generators. It is recommended to qualify equipment intended for installation in these areas for higher magnetic field immunity levels (RS101 and corresponding IEC EN61000-4-8 [11], IEC EN61000-4-9 [12], and IEC EN61000-4-10 [13] tests). It is important to pay attention to the selection of the enclosure materials for the equipment installed in this area, and use ferromagnetic materials where possible. Zones of increased E- and $\mathrm{H}$-field emissions should be physically marked in the plant.

\section{Methodology Inputs}

This chapter describes the preparation, carrying out, and potential usage of in situ immunity tests and site survey measurements as well as the challenges associated with the use of numerical calculations as a part of the EMC room classification methodology. All these methodology inputs are supported by actual examples from the plant.

\subsection{In Situ Immunity Tests}

The most complex activity, and presumably the most valuable input of the presented methodology, are the in situ immunity tests. In situ immunity tests can be performed almost exclusively during the outage, when the impact to plant operation is minimized. For the same reason, it is further recommended to limit these tests to the core offload window only. This limit could possibly interfere with the requirement that the equipment must be energized and have meaningful (nominal if possible) indications that can be observed. In addition, it is advisable to perform tests during the night shifts, not only for the above presented reasons, but also to be confident with a greater level of certainty that the equipment response is a result of the intentional electromagnetic and radio frequency interference (EMI/RFI), and not some unrelated activity [14].

The first task is to decide which equipment should be tested. It should be based on the project motives, industry operational experience, importance of the equipment for plant safety and power production, estimation of the equipment susceptibility and severity of its EME, quantity of similar or identical components in the plant, and other factors. The total number of equipment must be limited due to the substantial duration of each test. The rooms in which the most important I\&C equipment is located such as the main control room (MCR), emergency control room (ECR), computer rooms, etc. were analyzed more thoroughly by choosing a larger number of the cabinets and other equipment for the immunity tests. Other than nuclear safety-related equipment, special attention was given to the systems important for power production such as the main generator. For these systems, usually the main control cabinets and the most important instrumentation are tested. In total, in the case of the Krško NPP assessment, 47 different cabinets and instruments were selected. It is important to add that some equipment such as process cabinets consist of many subcomponents that were also encompassed in these tests.

The monitored outputs for the equipment under test can be defined arbitrarily, but they mostly depend on the significance of the particular outputs. In the simple case of a pressure transmitter, it is reasonable to monitor the pressure value locally or remotely. It is less straightforward as to what should be observed in the case of complex process cabinets, but usually the choice is reduced to the local indications and displays, alarms, and different parameters available on the plant computer and/or $\mathrm{MCR}$ recorders. The acceptance criteria can also be arbitrarily defined (e.g., a predefined percentage of the process range for the monitored signals).

The injected EMI/RFI test levels usually correspond to those defined for the MIL-STD-461 RS103 test (i.e., $10 \mathrm{~V} / \mathrm{m}$ over the whole frequency range), which provides at least an $8 \mathrm{~dB}$ margin above the $4 \mathrm{~V} / \mathrm{m}$ transceiver emissions limit. However, it is possible to also apply higher levels, in order to 
minimize the test uncertainty, or to prove that it is possible to use different devices, like plant radios or tablets, within a closer distance of cabinets and other equipment.

In situ immunity tests are intended to determine the response of the tested equipment, while not affecting other equipment located in the vicinity of the equipment under test (EUT). Thus, they are limited only to the radiated immunity tests. The radiated noise can be localized more easily when compared to the conducted noise (also, some conductive immunity tests are defined as destructive). In order to do this, it is possible to reduce the distance between the emitting antenna and EUT, thereby reducing the effective radiated area of the EUT (sometimes this is inevitable because of a lack of space), or simply by shielding the other equipment in the vicinity. The latter should include the equipment behind the antenna, as some antennas could have radiation patterns with significant back lobes. Shielding can be achieved by using copper nickel or similar fabric, which offers good shielding effectiveness (SE) up to the highest frequencies of interest. If it is recognized that the other equipment could cause any unwanted actuation, it should be disabled by putting it in test mode or by directly disabling executive components (e.g., putting pump switches to pull-out positions).

The frequency range of in situ immunity tests depends on the plant motives. It could be optimized for troubleshooting the problems with plant radios, in which case, the focus will be on the $400-500 \mathrm{MHz}$ range. If the plant wants to examine the implementation of new wireless technologies, it will more closely study higher frequencies. The most common wireless data standards include Bluetooth and $\mathrm{Wi}-\mathrm{Fi}$, where it is important that both the existing equipment is not vulnerable in those frequency ranges, and that all of the equipment that utilizes wireless technology can coexist in the same frequency band.

Different wireless technologies are characterized by different signal modulations. In order to optimize the test time, which can be crucial for the realization of the tests during the short system windows, it is practical to use conservative modulation schemes taken from military EMC tests. It can be assumed that it is more severe than other typically used modulations. The RS103 test uses a $1 \mathrm{kHz}$ pulse modulation and 50\% duty cycle, where the fast rise and fall time of the pulse produces significant harmonic content that affects analog and digital circuits. The more detailed explanation of why this modulation was chosen can be found in MIL STD 461G [15]. A typical test setup is shown in Figure 3 and shows an immunity test setup for a plant protection system cabinet, which consists of several signal processing cards, using a vertically-polarized log-periodic antenna.

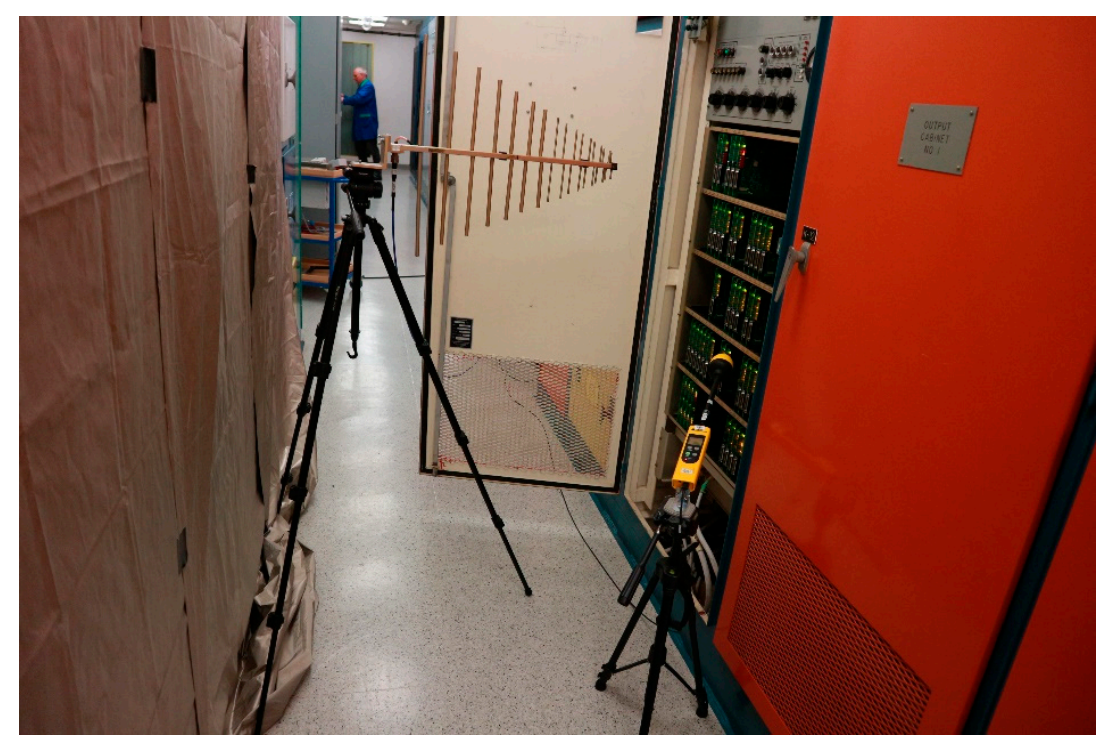

Figure 3. Immunity test of a protection system cabinet in the main control room.

Figure 4 shows the responses of one of the cabinet's signal processing cards captured by the plant computer for four different configurations (open and closed doors and both polarizations). As expected, the largest signal deviation was obtained with the open doors and vertically polarized 
antenna. Other polarizations between the horizontal and vertical one can also be used, especially if it is dictated by the layout of the tested components such as cables within the cabinet. By selecting only frequencies of interest, worst-case polarizations and modulations, reducing the dwell time (time at which the interfering signal is injected for a particular frequency), and by testing more components at the same time, it is possible to significantly reduce the total test duration (from a couple of hours to approximately $30-40 \mathrm{~min}$ per test), and to test more equipment in a given period without making any significant compromise on trustworthiness of the results. The minimum dwell time is limited by the response time of the equipment and sampling time of the recording system (local recorder, plant computer, or other). Additionally, the test should be automated by using a single signal generator and predefining the test frequencies. In this case, it is only necessary to change the emitting antennas, depending on the frequency range, and change their polarization.

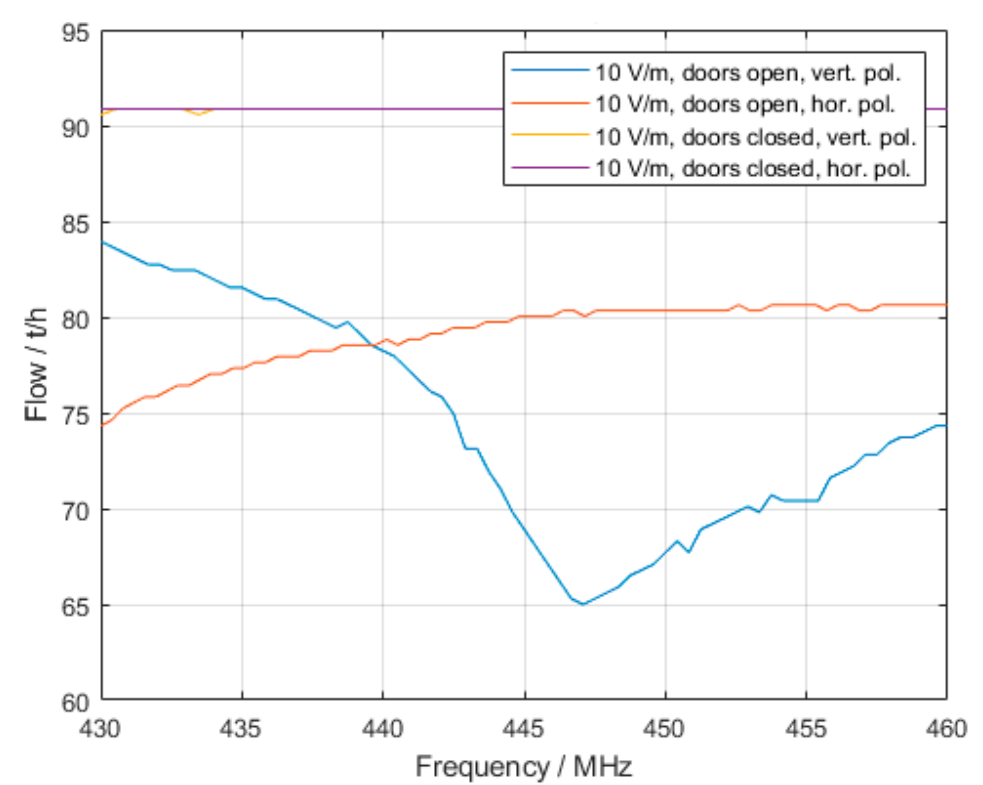

Figure 4. Signal processing card response for different test wave polarizations and open/closed cabinet doors.

In case the susceptibility is identified, several options are possible. If the equipment is conservatively tested with the doors open, or without its enclosure in general, it is possible to retest it in a less vulnerable configuration, which is usually the default one. Another option is to modify the equipment in order to improve its immunity. This includes the use of additional shielding, gaskets, EMC cable glands, ferrites, filters, and other methods. Figure 5 shows the response of a pressure transmitter in the original configuration compared to the one with the improved shielding. The EUT is susceptible to a relatively narrow band of frequencies around $780 \mathrm{MHz}$ due to a discontinuity in cable shielding in the flexible portion of the conduit. The problem can be solved by using a metallic cloth over the flexible conduit and assuring that it has good metal-to-metal contact on both its ends with the solid conduit as well as the transmitter enclosure. If none of these measures is effective, it is always possible to define an exclusion zone around the susceptible equipment. 

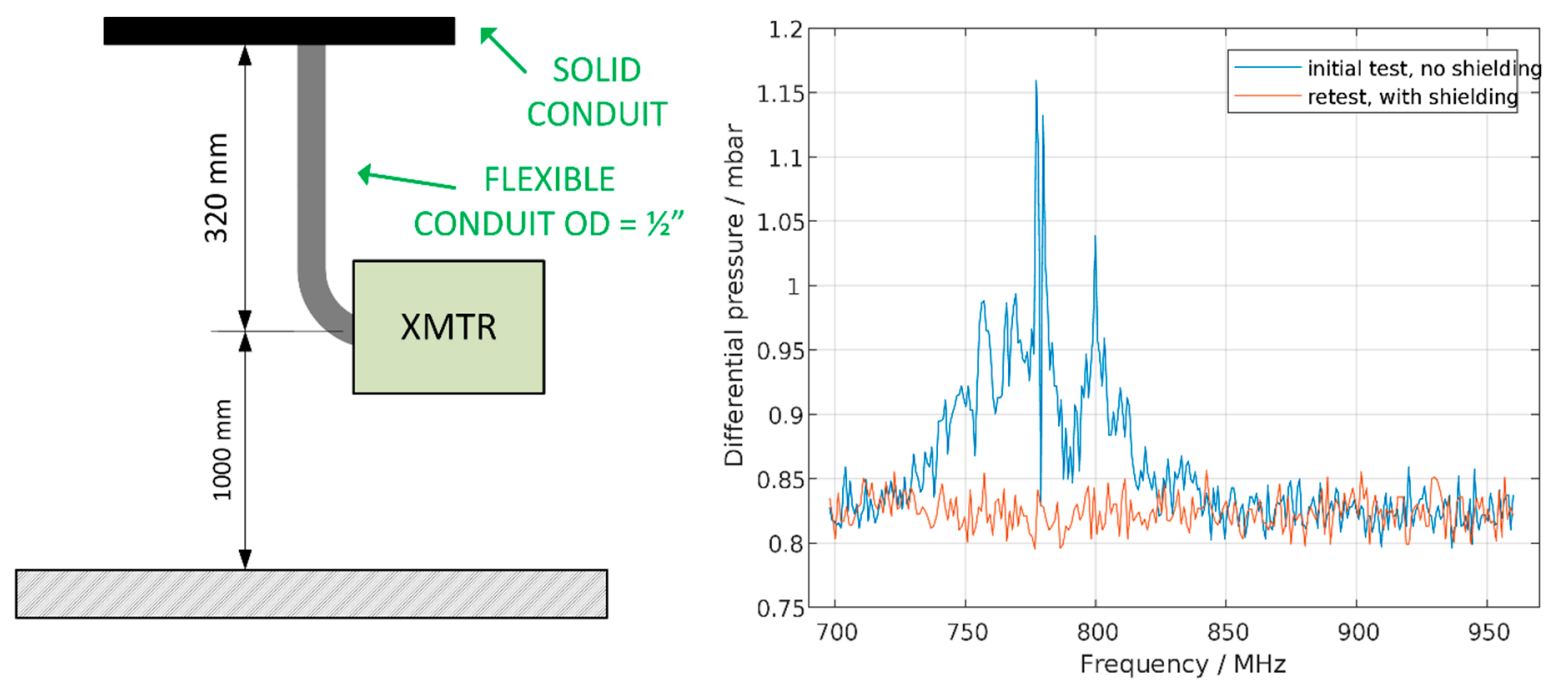

Figure 5. Transmitter installation detail and response of the measured output to EM noise with and without additional shielding.

One of the most important advantages of these tests, compared to the EMC qualification tests, is that they reflect the authentic installation, whereas qualification tests only try to replicate it. Most of the identified problems can be attributed to a few major sources. These are usually related to inadequate installations and obvious EMC design flaws. Several independent immunity tests have confirmed this statement. The exposed cables and inappropriately shielded equipment can easily pick up the EMI/RFI noise from the environment.

\subsection{Site Survey Measurements}

Site survey, sometimes also referred to as zone mapping, is a passive measurement of the EME levels in a power plant. In situ tests help to understand how the EM noise characteristics such as their amplitude, frequency, and bandwidth correlate with the EMI and risk to plant operation [16]. It is not reasonable nor possible to perform this in all plant areas. The areas of interest include rooms where the susceptible I\&C equipment is located as well as areas with potentially excessive EM emissions. In addition, it is important to include areas where the related plant modifications, especially digital upgrades, are foreseen. The tests are based on emission tests described in MIL-STD-461 (CE101, CE102, RE101, and RE102) and the corresponding commercial standards, however, they were slightly modified to fit the site survey purpose.

In contrast to the in situ immunity tests, site survey should be performed during the on-line operation. Otherwise, during outage, a large number of equipment are de-energized and do not contribute to the plant EM emission levels.

The plant EME is never in a steady state. Infrequent EM phenomena and/or transients could appear during plant startup, surveillance tests, and while starting significant loads. Thus, site survey should be scheduled simultaneously with these activities. These kinds of measurements are always challenging to perform. Measurement of the plant emissions in different frequency ranges requires the use of different measuring equipment, in particular, various antennas. The main reason is that different antennas have different gain profiles across different frequency ranges. Often, it is necessary to decide which phenomena should be recorded during the short duration of the transients. Some (un)intentional emitters, which are expected to be used in the plant such as plant radios and welding machines, can also be easily included in this survey.

Radiated emissions are preferably made with two opposite antenna directions. First, in the direction away from the equipment, the emissions are measured as seen by a cabinet or an instrument. Second, the measurements are taken in the opposite direction toward the equipment, in order to 
measure the emissions of the same equipment. In both cases, it should be considered that there is a background emission level (i.e., the hypothetical cabinet is not the only contributor).

Site surveys are not strictly related to radiative emissions. It is possible to also obtain conductive emissions in an unobtrusive way by using current clamps. Transient phenomena should be captured in the time domain, in contrast to the other measurements for which frequency domain is more suitable.

Although it could be said that each new modification affects the plant EME, it is not the intention to repeat this assessment too often, except in the case of major changes.

The output of this assessment are the emission data in the form of the amplitude vs. frequency graphs, as captured by the electric field antenna and a computer-controlled spectrum analyzer in peak-hold mode and as shown in Figure 6. The electric field is given in $\mathrm{dB}$ as a ratio of the amplitude to the specific reference, which in this case is $1 \mu \mathrm{V} / \mathrm{m}$. To assess the severity of the measured EME, the results were compared to the equipment emission limits for RE102 as defined by the standards shown. Another possibility is to compare the EM spectrum recorded in one room to the spectrum recorded in the reference room, in our case the main control room.

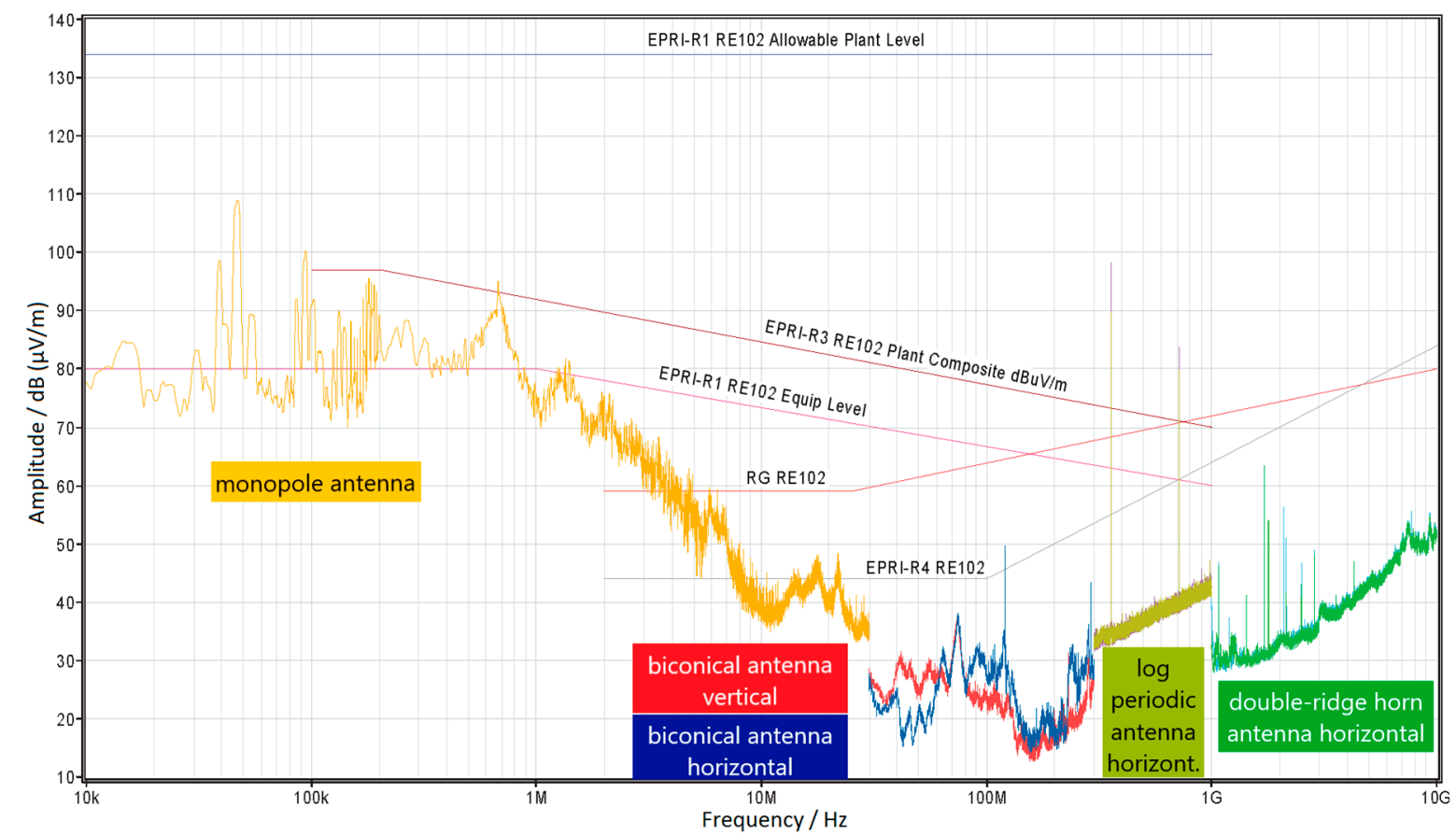

Figure 6. RE102 emissions and regulatory limits for one specific plant room (combined emissions captured by three antennas).

\subsection{Numerical Calculations}

Numerical calculations are not widely used for assessments of this type, nor for the complex systems and EMEs in general. Usually, their use is limited to the more simple EMC problems in the product design phase where they help to predict the emission and immunity properties of the newly developed equipment. The main reason for the limited use of the numerical models for the larger systems is the complexity of modeling realistic plant systems, but there are also limitations of the available software solutions.

As mentioned, it would be impractical and time consuming to perform measurements for a large number of discrete locations in order to obtain field distribution data. Instead, a model of the EM source and surrounding environment can provide more useful and meaningful data. It can also be used to compare different design variations (material properties, aperture size, position and shape, equipment orientation, etc.) and operational characteristics prior to equipment fabrication or installation. 
For this type of problem, the method of moments (MoM) is usually used. It is a full-wave solution of the Maxwell integral equations in the frequency domain [17]. Its advantage is that only the structure (wires, enclosures, etc.) are discretized, while the free space is not. Boundary conditions do not have to be set. As can be clearly seen in the next example, memory requirements depend on the complexity of geometry and the frequency of the solution. MoM is usually coupled with several extensions that allow for the modeling of magnetic and dielectric media.

An example of a typical plant cabinet was analyzed. The components were placed in a cabinet made of commercial steel, with approximate dimensions of $864 \times 1245 \times 1981 \mathrm{~mm}(\mathrm{w} \times \mathrm{d} \times \mathrm{h})$. On the front side are two swing doors, which represent the largest aperture of the cabinet. The gap between the doors and the cabinet has caused immunity problems during electrical field susceptibility tests. Another EMC problem that can be expected is due to several instruments mounted on the front doors, which present enclosure discontinuities. On top of the cabinet, there are two large fan openings as well as AC and DC cable entrances. On the bottom of the cabinet is a grill that serves as an air inlet. The side and back plates are fixed to the cabinet base.

The shielding effectiveness of the enclosure was degraded due to the mentioned apertures. The simulation was performed for different configurations of the front side of the enclosure to analyze the simplified cabinet properties and to explore software capabilities in real applications. The intention was not to model the tested cabinet and observe all related phenomena, but to demonstrate some of the opportunities that numerical calculations offer. Two different types of problem were studied for the same cabinet. The first, where the noise source is located inside the enclosure, and the second, where the noise is an external plane wave and the receptor is within the enclosure.

The radiating source within the cabinet is represented by wires in four different configurations, with one or more horizontal and vertical wires. Usually, calculations are performed for the range of source frequencies. The obtained value is the near-field electric field amplitude. For the lowest frequency of the range, $2 \mathrm{MHz}$ (lower limit of the RE101 test), the distribution of the electrical field amplitude $(\mu \mathrm{V} / \mathrm{m})$, in $\mathrm{YZ}$ planes at 10 and $100 \mathrm{~cm}$ from the front of the cabinet, is shown in Figure 7.

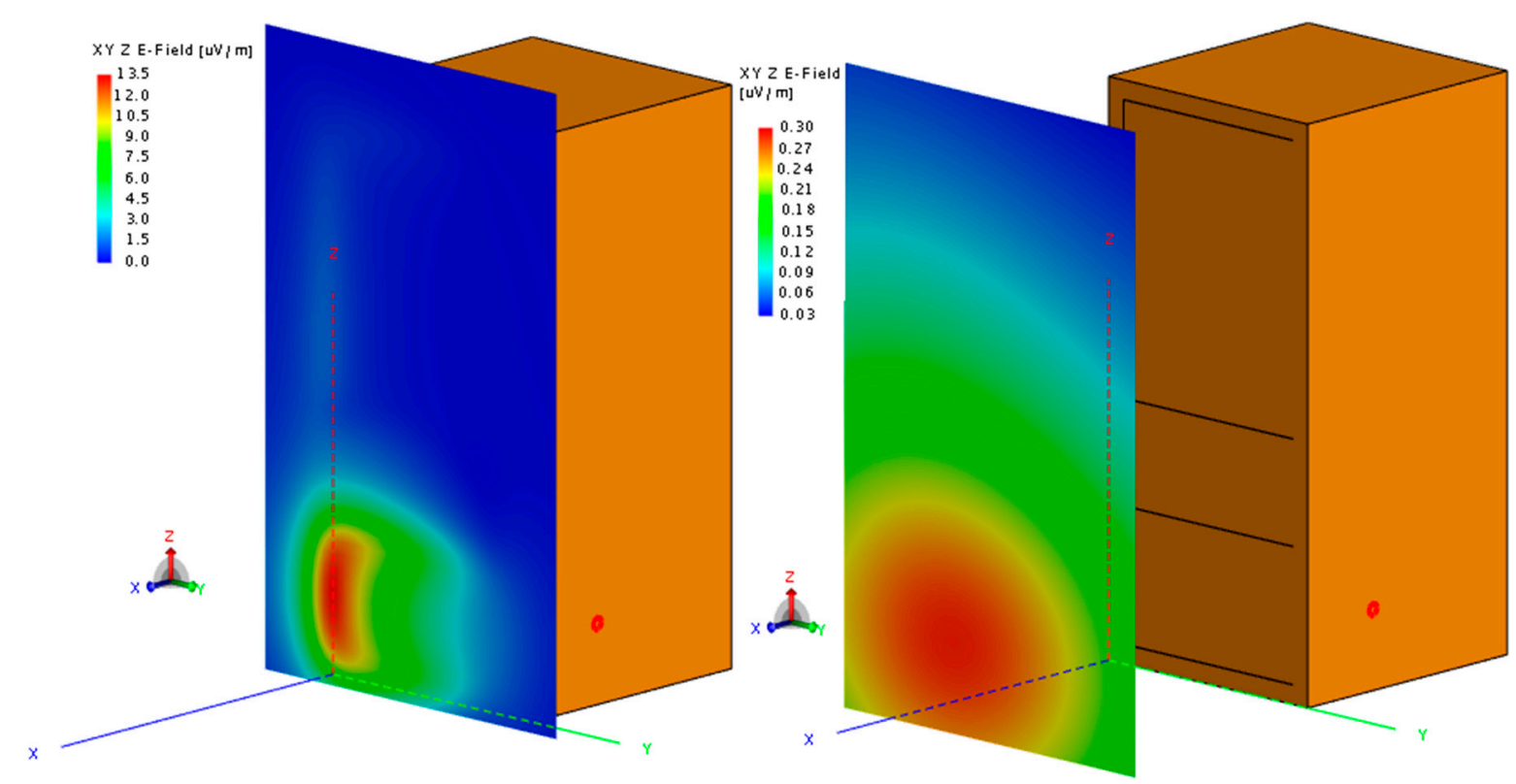

Figure 7. E-field values $(\mu \mathrm{V} / \mathrm{m}) 10 \mathrm{~cm}$ (left) and $100 \mathrm{~cm}$ (right) from the cabinet doors, at $2 \mathrm{MHz}$, noise source modeled as a horizontal wire in the lower cabinet part.

The results can provide additional information regarding the electric and magnetic field distribution in any plane parallel to the cabinet sides for different distances from them. The obtained trends are in accordance with the measured data. The simulation could be easily repeated for diverse cabinet 
designs to see impacts of the enclosure material and thickness, aperture size, shape and position on the field outside the cabinet.

In addition, E-field shielding effectiveness, in the case of the external plane wave, for two different cabinet layouts was compared: the default case with two cabinet doors, and case(s) with the lower doors only. Figure 8 shows that the additional door does not only degrade the overall SE of the cabinet, but can also introduce an additional resonant frequency. The results are given inside the cabinet, in the middle of the lower and upper door and in the middle of the area between doors. As expected, the higher the radiating source frequency, the less effective the enclosure.

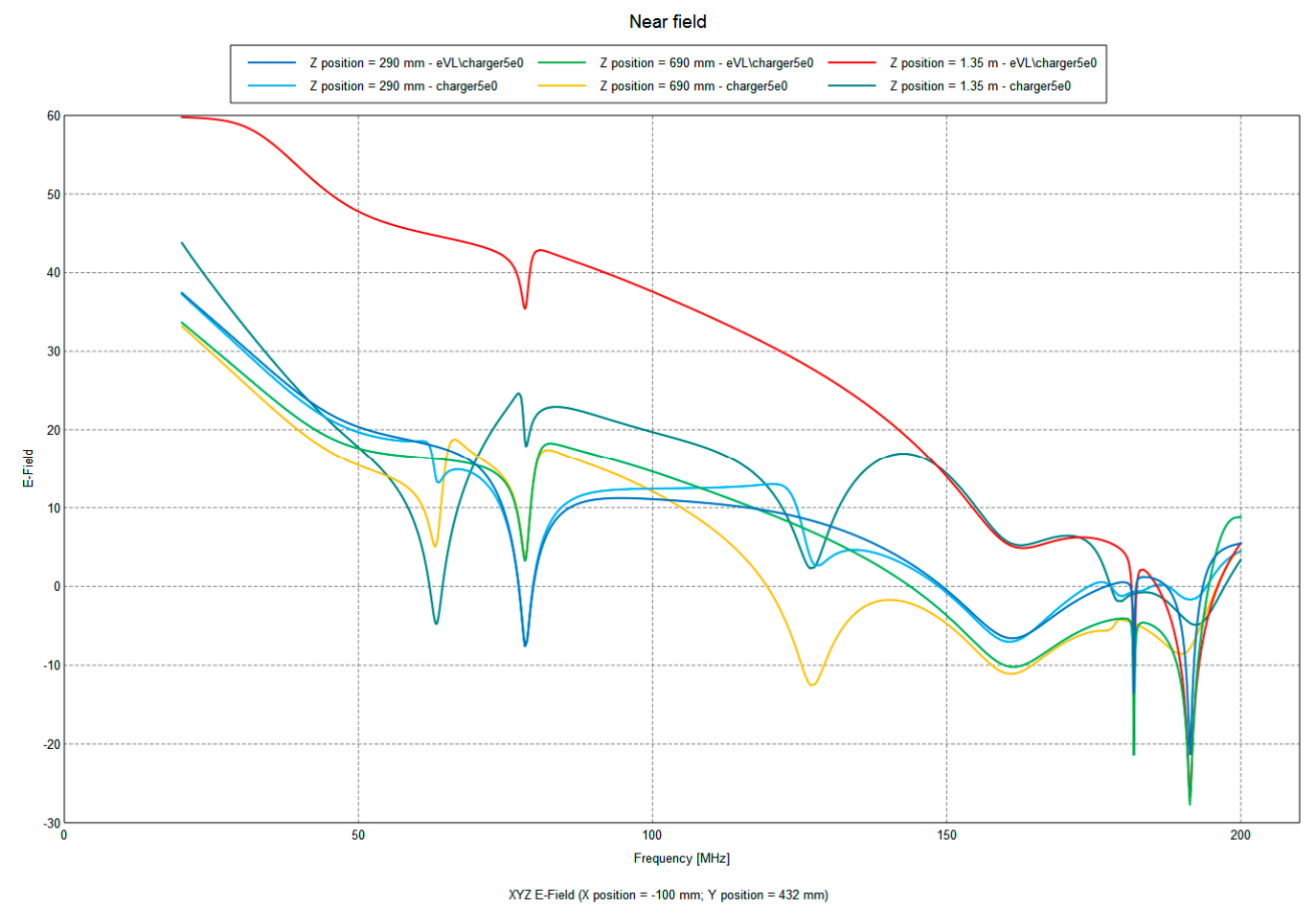

Figure 8. E-field shielding effectiveness $(\mathrm{dB})$ for two different cabinet layouts (lower door only and both doors) and for three axial positions at $10 \mathrm{~cm}$ inside the cabinet (vertical plane polarization).

The results obtained with the MoM confirm the significance of the openings on the shielding effectiveness. Some of the improvements such as the minimization of the door gap size can be easily quantified. Clearly, some design details, for example, ventilation openings, must be optimized for more parameters than only the EMC.

The computational time and memory consumption (several hours and between 12 and $120 \mathrm{~GB}$ ) increase with the dimensions of the model, level of details (in this case determined by door gap size), and observed frequency range. This could be a serious limitation for the calculation of real plant equipment.

\section{Zone Classification Applied to the Main Control Room}

An example of a typical MCR with several different EM attributes is shown in Figure 9. It describes that room from the EMC perspective, and includes information about the susceptibility and emission properties. The electromagnetic environment of the MCR was described with the set of parameters given in Table 2. 


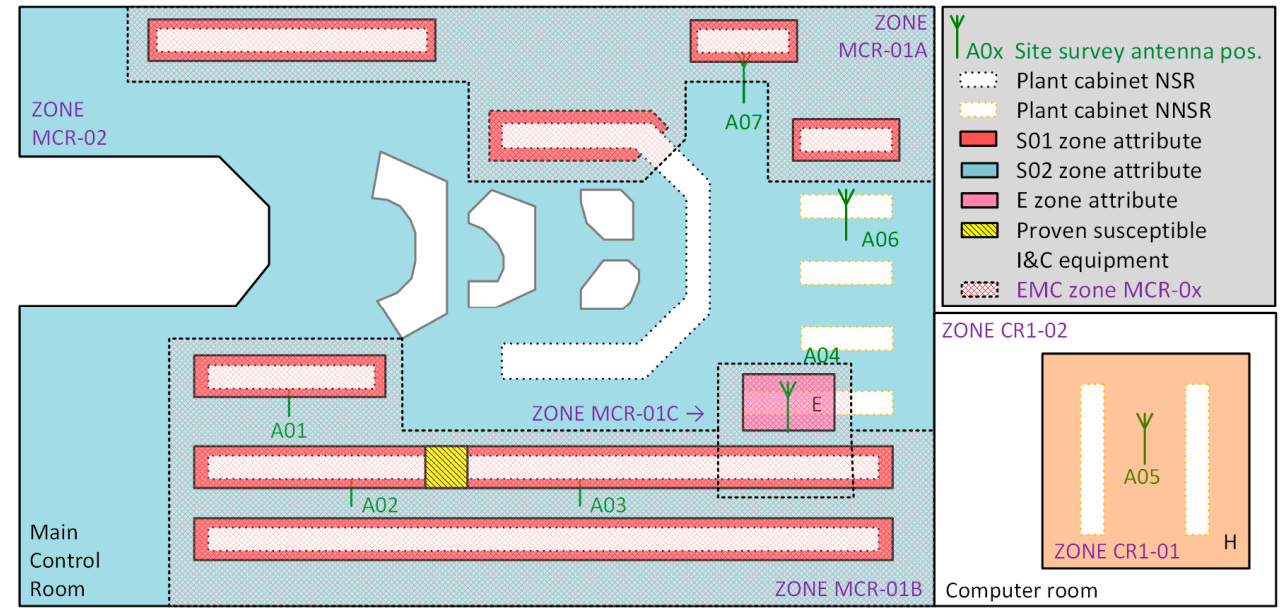

Figure 9. Example of the zone classification for a typical MCR.

Table 2. Zone MCR-01A EMC parameters.

\begin{tabular}{|c|c|c|}
\hline$\#$ & Parameter & Value \\
\hline 1 & Zone name & MCR-01A \\
\hline 2 & $\begin{array}{c}\text { Location } \\
\text { ID/Building/Elevation }\end{array}$ & Main Control Room 01 \\
\hline 3 & Zone attributes & $\mathrm{E} / \mathrm{H}$ attribute: none \\
\hline 4 & Dominant field type & $\begin{array}{l}\text { More severe magnetic fields; equipment susceptibility more emphasized for } \\
\text { electric fields (for E-field noise of several hundred MHz) }\end{array}$ \\
\hline 5 & Propagation path & Both radiative and conductive \\
\hline 6 & Emission spectrum & $\begin{array}{l}\text { Both narrowband (10 kHz, see below) and broadband ( } 20 \mathrm{kHz} \text {, unknown } \\
\text { source) }\end{array}$ \\
\hline 7 & $\begin{array}{l}\text { Emission } \\
\text { frequency/amplitude }\end{array}$ & $\begin{array}{l}\text { Mild; below EPRI/RG envelopes (RE101 at least } 20 \mathrm{dBpT} \text { and RE102 at least } 30 \\
\mathrm{~dB}(\mu \mathrm{V} / \mathrm{m}) \text { below EPRI allowable plant levels;); below plant average; } \\
\text { noticeable RE101 emissions at } 10 \mathrm{kHz} \text { (fluorescent lighting tubes); low UPS } \\
\text { emissions up to } 1 \mathrm{kHz} \text {; low level broadband noise with no narrowband } \\
\text { transmitters or specific noise sources above } 30 \mathrm{MHz} \text { (RE102) }\end{array}$ \\
\hline 8 & Emission duration & Continuous during online operation; intermittent during outage \\
\hline $8 \mathrm{a}$ & $\begin{array}{l}\text { Intermittent } \\
\text { occurrence rate }\end{array}$ & Low; dependent on operators' switching operations \\
\hline 9 & Source nature & $\begin{array}{l}\text { Man-made; protected against outside natural effects (such as lightning strikes) } \\
\text { as it is an inner plant area }\end{array}$ \\
\hline $9 a$ & $\begin{array}{l}\text { Man-made source } \\
\text { nature }\end{array}$ & Unintentional (no intentional interference as it is an exclusion zone) \\
\hline 10 & Immunity tests & $\begin{array}{l}\text { Passed without any modifications. Only general immunity improvements } \\
\text { discussed for S01 are recommended. }\end{array}$ \\
\hline 10a & $\begin{array}{l}\text { Exclusion zone } \\
\text { distance }\end{array}$ & $\begin{array}{l}\text { Calculated exclusion distances below } 2 \mathrm{~m} \text { for all combinations of EUTs and } \\
\text { possible emitters. }\end{array}$ \\
\hline 11 & Note & $\begin{array}{l}\text { No outstanding emissions except fluorescent lighting. MCR is intensely } \\
\text { interconnected with the rest of the plant }\end{array}$ \\
\hline
\end{tabular}

The nuclear safety-related cabinets, especially those found susceptible to EMI, are the most critical components. In this example, one part of the main control board (MCB), which is replaced with a digital system, is also described with $\mathrm{S} 01$ attributes, in contrast with the rest of the board, which mainly consists of the EM unsusceptible hardwired controls and indications. A special zone MCR-01C was defined for the area with both increased emissions (mostly produced by UPS racks) and susceptible equipment. The same figure also shows the exact locations of the site survey measurements and I\&C equipment, for which it is known that it did not pass the in situ immunity tests. MCR-01A/B zones, 
described with the $\mathrm{S} 01$ attribute, encompass a distance up to two meters from all nuclear safety-related plant cabinets and I\&C instrumentation. The MCR-01C zone is based on the increased E-field emissions, and the boundaries are determined based on the site survey measured emissions and calculated field attenuation according to Equation (1). In this case, the zone would encompass two plant cabinets. The rest of the MCR, designated as zone MCR-02, contains NNSR cabinets, which were found to be immune for all generated upsets. This zone is most accurately described with S04 attributes, which is a major relaxation when compared to the traditional practices, and is an important point for the potential implementation of wireless technologies.

\section{Conclusions}

The EMC requirements for nuclear power plants have become more important as the analogue I\&C equipment becomes obsolete, and is being replaced with a digital system. Furthermore, there is a tendency to make more use of wireless technologies, and the EM environment is consequentially rapidly changing and becoming more complex. In case of the Krško NPP, the EMC classification was initiated by problems with the sensitivity of the protection system replacement card to the operation of the switch on the main board. Even though the EM environment in the control room was not changed, the new design of the protection system card (limited availability of old cards) caused the plant to trip. Less severe problems were experienced with new digital systems introduced at the plant as a replacement for old analog systems.

Nuclear power plants are technically complex facilities and are therefore extremely challenging to analyze from the EMC point of view. The paper describes a methodology to address the EMC problem in a systematic way. The methodology was based on the experience from recent efforts made at the Krško NPP to develop/implement a novel EMC program at the plant. The results obtained in the initial survey showed the importance of a systematic approach and were used to develop a methodology in an iterative way (the results were used to develop the methodology, and vice versa, the methodology was reapplied to obtain more complete data).

The performed work includes the selection of the plant locations for the EMC assessment, the execution of the comprehensive in situ emission measurements and immunity tests, numerical modeling to extrapolate unavailable data, synthesis of different input data, evaluation of such results from the operational point of view (whether the proposed measures, in particular, exclusion zones, are too strict for the normal plant activities, or if on the contrary, they present a risk for the plant operation), and finally the classification of the plant areas.

The measurement results obtained from the qualification tests provide limited information regarding the equipment EM emissions. Still, they are the only regulatory requirement. Operating experience records, site survey results, in situ and bench immunity test results, and numerical calculations can provide additional information to help us understand, quantify, and improve the EMC level of the plant. Numerical calculations have large potential, which is mostly neglected, except partially in the equipment design stage. Even simplified models can explain some of the phenomena observed during the qualification tests and other measurements. The importance of such information is even greater emphasized, considering that the older equipment often lacks any data regarding its EM emissions and immunity.

The developed methodology, in contrast to the traditional approach based on generic EMC equipment qualification in nuclear power plants, classifies plant areas into a reasonable number of EMC zones. Each zone is described with similar EMC attributes, and is based on the various input data, which include practical test measurements as well as numerical models. This methodology defines the basic requirements for the installation of the new equipment and replacement of the existing as well as rules for the exclusion zones. The outcome of the methodology is highly dependent on the quality and quantity of the input data. The more comprehensive data could lead to the use of more quantitative and less qualitative criteria. Some steps such as site surveys can be performed in significantly different 
ways. This paper outlines the most important steps of each assessment, thereby assuring repeatability and reproducibility of the results despite the general lack of such guidance.

The initial methodology results have confirmed that the EMC room classification has helped to unify plant EMEs. These were also used to fulfill regulatory body requests for more systematic analysis of the EM conditions in the plant. The resulting zones were more complex compared to the EQ classification, just as expected. An example of the obtained data for the EMC zone is given for the main control room. Some of the data are approximate due to proprietary reasons.

The resulting exclusion zones were larger compared to the EPRI general recommendation of $1 / 3 \mathrm{~m}$, yet smaller for many equipment, for which, very conservatively, large distances were previously used (sometimes even whole plant rooms). This significant result ensures both equipment invulnerability and the flexibility of the plant personnel.

The methodology presented in this paper is general enough and can be applicable to different power plants. However, in the case of changes in the EME, caused by the introduction of new technologies and digital upgrades, the existing results may be affected and it could be necessary to reapply the methodology. Therefore, maintenance of the EMC in the plants will remain an ongoing activity and challenge.

Author Contributions: Conceptualization, H.G. and D.G.; Methodology, H.G.; Software, D.G. and S.̌̌.; Validation, H.G.; Formal analysis, H.G.; Investigation, H.G..; Data curation, H.G. and S.Š.; Writing-original draft preparation, H.G.; Writing-review and editing, D.G. and S.̌̌.; Visualization, H.G. and D.G.; Supervision, D.G. All authors have read and agreed to the published version of the manuscript.

Funding: This research received no external funding.

Acknowledgments: The authors express their gratitude to the Krško NPP for providing the results of the EMC assessment.

Conflicts of Interest: The authors declare no conflicts of interest.

\section{References}

1. Kercel, S.W.; Korsah, K.; Wood, R.T. Susceptibility of digital instrumentation and control systems to disruption by electromagnetic interference. In Proceedings of the International Topical Meeting on Advanced Reactors Safety, Orlando, FL, USA, 1-5 June 1997.

2. Electromagnetic Compatibility (EMC)-Part 2-5: Environment Description and Classification of Electromagnetic Environments; IEC TR 61000-2-5:2017; IEC: Geneva, Switzerland, 2017.

3. Lowe, C.L.; Kiger, C.J. Using a Cognitive Radio System to Support Wireless Technology Implementation at Nine Mile Point. In Proceedings of the 61st ISA Annual Power Industry Division (POWID) Symposium, Knoxville, TN, USA, 26-28 June 2018.

4. Lowe, C.L.; Kiger, C.J. In-Situ Electromagnetic Immunity Testing of Nuclear Power Plant Equipment. In Proceedings of the 58th Annual ISA POWID Symposium, Kansas City, MO, USA, 7-11 June 2015.

5. Lowe, C.L.; Kiger, C.J.; Jackson, D.N.; Young, D.M. Implementation of Wireless Technologies in Nuclear Power Plants' Electromagnetic Environment Using Cognitive Radio System. In Proceedings of the American Nuclear Society 10th International Topical Meeting on Nuclear Plant Instrumentation, Control and Human-Machine Interface Technologies (NPIC \& HMIT), San Francisco, CA, USA, 11-15 June 2017.

6. Kercel, S.W.; Moore, M.R.; Blakeman, E.D.; Ewing, P.D.; Wood, R.T. Survey of Ambient Electromagnetic and Radio-Frequency Interference Levels in Nuclear Power Plants; No. NUREG/CR-6436; Nuclear Regulatory Commission: Rockville, MD, USA, 1996.

7. International Atomic Energy Agency. Equipment Qualification in Operational Nuclear Power Plants: Upgrading, Preserving and Reviewing; Safety Reports Series No. 3; IAEA: Vienna, Austria, 1998.

8. Barnes, P.R.; Manweiler, R.W.; Davis, R.R. The Effects of Nuclear Electromagnetic Pulse (EMP) on Nuclear Power Plants; ORNL-5029; Oak Ridge National Lab.: Oak Ridge, TN, USA, 1977.

9. Electric Power Research Institute (EPRI). Guidelines for Electromagnetic Compatibility Testing of Power Plant Equipment; TR-102323 rev.4; EPRI: Palo Alto, CA, USA, 2013. 
10. Electromagnetic Compatibility (EMC)-Part 4-3: Testing and Measurement Techniques-Radiated, Radio-Frequency, Electromagnetic Field Immunity Test; IEC 61000-4-3:2006; IEC: Geneva, Switzerland, 2016.

11. Electromagnetic Compatibility (EMC)-Part 4-8: Testing and Measurement Techniques-Power Frequency; IEC 61000-4-8:2009; IEC: Geneva, Switzerland, 2009.

12. Electromagnetic Compatibility (EMC)-Part 4-9: Testing and Measurement Techniques-Pulse Magnetic Field Immunity Test; IEC 61000-4-9:2016; IEC: Geneva, Switzerland, 2016.

13. Electromagnetic compatibility (EMC)-Part 4-10: Testing and Measurement Techniques-Damped Oscillatory Magnetic Field Immunity Test; IEC 61000-4-10:2016; IEC: Geneva, Switzerland, 2016.

14. Grganić, H.; Valjak, M.; Škorc, G.; Romac, L. Ensuring Electromagnetic Compatibility in Nuclear Power Plant beyond Equipment Qualification Tests. In Proceedings of the 12th International Conference of the Croatian Nuclear Society, Zadar, Croatia, 3-6 June 2018.

15. Requirements for the Control of Electromagnetic Interference Characteristics of Subsystems and Equipment; MIL-STD-461G; IEC: Geneva, Switzerland, 2015.

16. Kiger, C.; Grganić, H. In-Situ Electromagnetic and Radio Frequency Interference Testing of Equipment in the Main Control Room of the Krško Nuclear Power Plant. In Proceedings of the American Nuclear Society 11th International Topical Meeting on Nuclear Plant Instrumentation, Control and Human-Machine Interface Technologies (NPIC \& HMIT), Orlando, FL, USA, 9-14 February 2019.

17. Mitin, V.V.; Sementsov, D.I. An Introduction to Applied Electromagnetics and Optics; CRC Press: Boca Raton, FL, USA, 2017; pp. 80-83.

(C) 2020 by the authors. Licensee MDPI, Basel, Switzerland. This article is an open access article distributed under the terms and conditions of the Creative Commons Attribution (CC BY) license (http://creativecommons.org/licenses/by/4.0/). 